\title{
Roles of microRNA-539 and osteopontin in rheumatoid arthritis
}

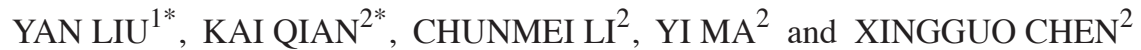 \\ ${ }^{1}$ Department of Rheumatology, Huai'an First People's Hospital, Nanjing Medical University, Huai'an, Jiangsu 223300; \\ ${ }^{2}$ Department of Rheumatology, Nanjing First Hospital, Nanjing Medical University, Nanjing, Jiangsu 210006, P.R. China
}

Received September 26, 2016; Accepted June 5, 2017

DOI: $10.3892 /$ etm.2017.5665

\begin{abstract}
The present study aimed to investigate the role and mechanism of microRNA-539 (miR-539) in rheumatoid arthritis (RA). A total of 68 RA patients and 46 osteoarthritis patients were enrolled into the current study. Peripheral blood and joint fluid were collected prior to treatment. Reverse transcription-quantitative polymerase chain reaction was performed to detect osteopontin (OPN) mRNA and miR-539 expression levels, while ELISA and western blot analysis were applied to detect OPN protein expression. In addition, bioinformatics analysis predicted that miR-539 directly targeted OPN, while dual-luciferase assay was used to validate this finding. Furthermore, agomiR-539 transfection and OPN knockdown by siRNA were conducted in MH7A cells, and MTT assay was used to detect MH7A cell proliferation. The results indicated that OPN was significantly increased in the blood and joint fluid of RA patients, while miR-539 expression was significantly decreased in the two types of specimens $(\mathrm{P}<0.05)$. Subsequent to silencing OPN by siRNA, the proliferation of MH7A cells was decreased $(\mathrm{P}<0.05)$. Following upregulation of miR-539, OPN expression was significantly decreased and cell proliferation was inhibited. Dual-luciferase assay revealed that miR-539 regulated OPN expression through complementary binding to 3 '-untranslated region. OPN was also significantly increased in the blood and joint fluid of RA patients, which may be associated with the downregulation of miR-539. Thus, miR-539 may promote the development and progression of RA through regulating OPN.
\end{abstract}

\section{Introduction}

Rheumatoid arthritis (RA) is a systemic autoimmune disease characterized by symmetrical arthritis, which may invade the

Correspondence to: Dr Xingguo Chen, Department of Rheumatology, Nanjing First Hospital, Nanjing Medical University, 68 Changle Road, Nanjing, Jiangsu 210006, P.R. China

E-mail: cheg333@126.com

*Contributed equally

Key words: microRNA-539, osteopontin, secreted phosphoprotein 1, rheumatoid arthritis heart, lung, kidney, artery, nerve and eyes $(1,2)$. The prevalence rate of RA ranks first among autoimmunity connective tissue diseases (3). The average incidence of RA is $0.5-1 \%$ worldwide, while it is $0.32-0.36 \%$ in China (4). RA can occur at any age with a high incidence rate, and the common age of incidence is 40-60 years. The majority of the RA patients are women, and the incidence rate is 2-3 times higher in comparison with that in men (5).

It has been reported that numerous microRNAs (miRs) participate in the development and progression of RA (6-8). In addition, miR-539 has been observed to be significantly upregulated in patients with heart failure (9), in the blood of patients with alcoholic hepatitis (10), and in human osteosarcoma cells (11). All these findings indicated that miR-539 is closely associated with the disease development. However, the role of miR-539 in RA remains unclear.

Osteopontin (OPN), also known as secreted phosphoprotein 1 or early T-lymphocyte activation 1 , is a potential promoter among inflammatory cytokines (12). A large numbers of OPN surface receptors are expressed in synovial cells, which combined with OPN to induce signaling transduction and influence the adhesion and proliferation of synovial cells (13). In RA and juvenile idiopathic arthritis patients, OPN protein has been reported to be significantly increased in the synovium, while it was also significantly higher in the articular cartilage of osteoarthritis patients, where it may induce the gradual degradation of articular cartilage (14). OPN protein in synovial fluid originates from the synovium and cartilage. Honsawek et al (15) observed that OPN protein expression was significantly increased in RA and osteoarthritis patients.

In the current study, OPN and miR-539 expression at the mRNA and protein levels were detected in the blood and joint fluid of RA patients. Next, the association between miR-539 and OPN expression was analyzed. The mechanisms underlying the role of miR-539 in the development and progression of RA were also discussed.

\section{Materials and methods}

Clinical data and sample collection. The present study included 68 RA patients, as well as 46 osteoarthritis patients serving as the controls, who were admitted between January 2014 and June 2015 to Nanjing First Hospital (Nanjing, China). Among the RA patients, 21 cases were male and 47 cases were female, with ages ranging between 26 and 72 years, and a median age of 51.6 years. The joint fluid was 
extracted from 11 (3 males and 8 females) out of the 68 RA patients, whose median age was 46 years. In the control group, 16 males and 30 females were included, with a median age of 53.5 years (range, 21-76 years old). Among these control cases, 9 patients (4 males and 5 females) were subjected to extraction of joint fluid, whose median age was 49 years. All the patients were diagnosed by pathologists in our hospital. The included patients presented the first onset of disease and had not received previous treatment, such as hormones, medication, radiotherapy or chemotherapy. Prior written informed consent was obtained from every patient, and the study was approved by the Ethics Review Board of Nanjing Medical University (Nanjing, China).

Fasting peripheral blood was collected from all patients on admission, and the serum was isolated from the samples by centrifugation at $1,000 \mathrm{x}$ for $10 \mathrm{~min}$. The joint fluid samples were collected by knee joint cavity paracentesis under a sterile environment by an experienced clinician and stored at $-80^{\circ} \mathrm{C}$ within $2 \mathrm{~h}$.

Reverse transcription-quantitative polymerase chain reaction (RT-qPCR). Total RNA was extracted from both serum and joint fluid by the TRIzol method (Shanghai Yisheng Biotechnology Co., Ltd., Shanghai, China), and RNA quality was examined by $10 \%$ SDS-PAGE and by determining the ratio of absorbance at 260/280 $\mathrm{nm}$ with a spectrophotometer. Total RNA was reverse transcribed into the cDNA for mRNA determination using miRcute miRNA First-Strand cDNA Synthesis kit (Tiangen Biotech Co., Ltd., Beijing, China), while the poly (A) tailing method was used to obtain miRNA cDNA, as described previously (16).

For RT, $1 \mu \mathrm{g}$ RNA was added to $2 \mu \mathrm{l}$ Oligo (dT), followed by addition of $\mathrm{H}_{2} \mathrm{O}$ to a final volume of $10 \mu \mathrm{l}$, and the mixture was centrifuged at $400 \mathrm{x} \mathrm{g}$ and $4^{\circ} \mathrm{C}$ for $5 \mathrm{sec}$. Following incubation at $72^{\circ} \mathrm{C}$ for $2 \mathrm{~min}$ and placing on ice for $2 \mathrm{~min}$, the mixture was centrifuged again at $400 \times \mathrm{g}$ and $4^{\circ} \mathrm{C}$ for $5 \mathrm{sec}$, followed by addition of $2 \mu \mathrm{l}$ dNTP Mix (10 mM), $2 \mu \mathrm{l}$ dithiothreitol $(20 \mathrm{mM}), 2 \mu \mathrm{l}$ Powerscript Reverse Transcriptase and $4 \mu 1$ 5X First-strand Buffer (Tiangen Biotech Co., Ltd.), and incubation at $42^{\circ} \mathrm{C}$ for $1.5 \mathrm{~h}$. The obtained cDNA was stored at $-20^{\circ} \mathrm{C}$. Subsequently, qPCR was conducted with the SYBR Green RT-qPCR kit (Tiangen Biotech Co., Ltd.) to detect the expression of OPN mRNA in the blood and joint fluid of patients. $\beta$-actin was used as an internal reference. Primers used in qPCR were as follows: OPN, 5'-GTTATGAAACGA GTCAGCTG-3' (forward) and 5'-TTAATTGACCTCAGA AGATG-3' (reverse); $\beta$-actin, 5'-AGCGGGAAATCGTGC GTG-3' (forward) and 5'-GAGGGTACATGGTGGTGCC-3' (reverse). The reaction was conducted in a $25-\mu 1$ system that included $10 \mu \mathrm{l}$ RT-qPCR-Mix, $0.5 \mu \mathrm{l}$ forward primer, $0.5 \mu \mathrm{l}$ reverse primer, $2 \mu \mathrm{l}$ cDNA template and $7 \mu \mathrm{lddH_{2 }} \mathrm{O}$. The cycling conditions were the following: Initial denaturation at $95^{\circ} \mathrm{C}$ for $5 \mathrm{~min}$, and 30 cycles of denaturation at $95^{\circ} \mathrm{C}$ for $30 \mathrm{sec}$, elongation at $58^{\circ} \mathrm{C}$ for $30 \mathrm{sec}$ and annealing at $72^{\circ} \mathrm{C}$ for $30 \mathrm{sec}$. The relative mRNA expression of OPN with respect to that of $\beta$-actin was calculated by the $2^{-\Delta \Delta \mathrm{Cq}}$ method (17).

The RT method used for miRNA followed the standard protocol provided by the manufacturer of the miRcute miRNA cDNA kit (Tiangen Biotech Co., Ltd.). This kit was used to detect the expression of miR-539 in the blood and joint fluid of patients. U6 was used as internal reference. The primers used in qPCR were as follows: U6, 5'-CTCGCTTCGGCA GCACA-3' (forward) and 5'-AACGCTTCACGAATTTGC GT-3' (reverse); miR-539, 5'-ACACTCCAGCTGGGATCA TACAAGGACAAT-3' (forward) and 5'-TGGTGTCGTGGA GTCG-3' (reverse). The $25 \mu \mathrm{l}$ reaction system included $10 \mu \mathrm{l}$ RT-qPCR-Mix, $0.5 \mu 1$ forward primer, $0.5 \mu 1$ reverse primer, $2 \mu \mathrm{l}$ cDNA template and $7 \mu \mathrm{l} \mathrm{ddH}_{2} \mathrm{O}$. The cycle conditions were the following: $95^{\circ} \mathrm{C}$ for $5 \mathrm{~min}$, followed by 40 cycles of $95^{\circ} \mathrm{C}$ for $15 \mathrm{sec}, 60^{\circ} \mathrm{C}$ for $30 \mathrm{sec}$ and $72^{\circ} \mathrm{C}$ for $30 \mathrm{sec}$. The relative expression of miR-539/U6 was calculated by the $2^{-\Delta \Delta \mathrm{Cq}}$ method.

ELISA. The serum and joint fluid samples were subjected to ELISA to determine the concentration of OPN, performed according to the manufacturer's protocol of the OPN ELISA kit (cat. no. ab100618; Abcam, Cambridge, UK). Briefly, $50 \mu 1$ of the standard samples at $0,74.07,222.2,666.7,2,000,6,000$ and $18,000 \mathrm{pg} / \mathrm{ml}$ were added into different wells on the ELISA plate. Next, $10 \mu \mathrm{l}$ sample and $40 \mu \mathrm{l}$ dilution solution was added into each well. With the exception of the blank well, each well was treated with $100 \mu \mathrm{l}$ biotinylated antibodies and incubated at $37^{\circ} \mathrm{C}$ for $1 \mathrm{~h}$. Subsequent to washing five times, $50 \mu 1$ substrate $\mathrm{A}$ and $50 \mu \mathrm{l}$ substrate $\mathrm{B}$ were added into each well, incubated at $37^{\circ} \mathrm{C}$ for $15 \mathrm{~min}$, and followed by addition of $50 \mu \mathrm{l}$ stop solution in order to terminate the reaction. Optical density values were measured at a wavelength of $450 \mathrm{~nm}$ within $15 \mathrm{~min}$.

Bioinformaticsprediction. Bioinformatics prediction is the basis to explore the functions of miRNAs, and this method was used in the present study to predict the potential miRNAs that regulate OPN directly. The miRanda (microrna.org/microrna/home. do), TargetScan (targetscan.org), PiTa (genie.weizmann. ac.il/pubs/mir07/mir07_data.html), RNAhybrid (bibiserv. techfak.uni-bielefeld.de/rnahybrid/) and PICTA (pictar. mdc-berlin.de/) software packages were used. According to the results of this analysis, miR-539 was selected to further exploring its association with OPN in RA progression.

Dual-luciferase assay. Based on the bioinformatics prediction, the wild-type 3'-untranslated region (UTR) (5'-AAU UUCUC-3') and the mutant 3'UTR (5'-UUAAAGAG-3') (both Sangon Biotech Co., Ltd., Shanghai, China) of OPN were synthesized in vitro and cloned into the Ambion ${ }^{\circledR}$ pMIR-REPORT ${ }^{\mathrm{TM}}$ miRNA Expression Reporter Vector system (Thermo Fisher Scientific, Inc., Waltham, MA, USA) by Spe-1 and HindIII restriction sites. 293T cells (Cell Bank, Chinese Academy of Sciences, Shanghai, China) were co-transfected with agomiR-539 (100 nM) mimics (5'-GGA GAAAUUAUCCUUGGUGUGU-3'; Sangon Biotech Co., Ltd.) and with the wild-type OPN 3'UTR or mutant 3'UTR using ExFect Transfection Reagent (Vazyme, Piscataway, NJ, USA). Subsequent to transfection for $24 \mathrm{~h}$, cells were lysed using the lysis buffer provided by the Dual-Luciferase Reporter Assay System kit (cat. no. E1910; Promega Corporation, Madison, WI, USA) and luciferase intensity was measured by a GloMax 20/20 luminometer (Promega Corporation) based on the standard manufacturer's protocol of the aforementioned kit. The intensity of Renilla was used 
A

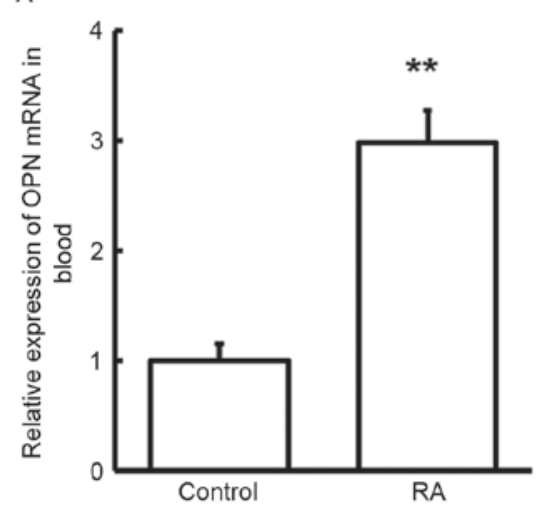

B

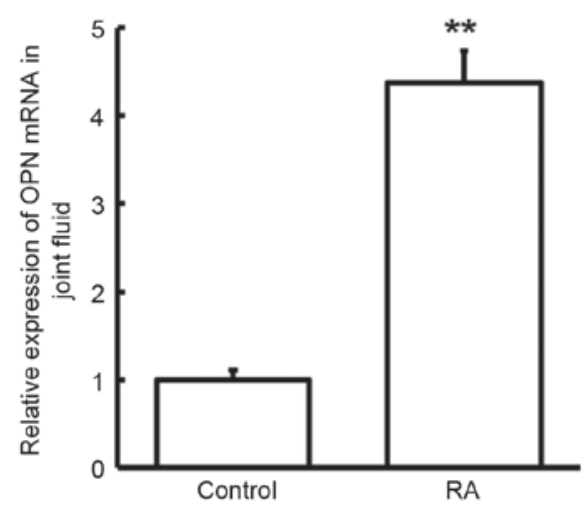

Figure 1. OPN mRNA expression in the (A) blood and (B) joint fluid of RA patients, determined by reverse transcription-quantitative polymerase chain reaction. Compared with the osteoarthritis (control) patients, the expression of OPN mRNA was significantly increased in the blood and joint fluid of RA patients. ${ }^{* *} \mathrm{P}<0.01$ vs. control patients. OPN, osteopontin; RA, rheumatoid arthritis.

as the control, and the fluorescence intensity in different groups was analyzed.

Cell transfection. At 1 day prior to transfection, $3 \times 10^{5}$ MH7A cells (Cell Bank, Chinese Academy of Sciences, Shanghai, China) were seeded into each well of a 24-well plate and cultured into antibiotic-free F12/Dulbecco's modified Eagle's medium (DMEM) containing 10\% fetal bovine serum (all Thermo Fisher Scientific, Inc.). When $\sim 70 \%$ confluence was reached, the cells were used for transfection. The pMIR-REPORT luciferase vector, OPN siRNA (5'-AAA CAGGCTGATTCTGGAAGTTC-3'; $50 \mathrm{nM}$ ), agomiR-539 (5'-GGAGAA AUUAUCCUUGGUGUGU-3'; $10 \mathrm{nM}$ ), agomiR-NC (5'-UUCUCCGAACGUGUCACGUTT-3'; 10 nM; all Sangon Biotech Co., Ltd.) and Lipofectamine 2000 (Invitrogen; Thermo Fisher Scientific, Inc.) were added into Eppendorf tubes containing $50 \mu \mathrm{l}$ DMEM. Next, the mixture was added to each well after incubating for $20 \mathrm{~min}$ at room temperature. Following transfection for $48 \mathrm{~h}$, the cells were collected to detect the expressions of OPN at the mRNA and protein levels.

Western blot analysis. Total proteins were extracted from MH7A cells based on standard protocol provided by the protein lysis kit (BestBio Science, Shanghai, China), and the protein concentration was detected by a BCA assay kit [Real-Times (Beijing) Biotechnology Co., Ltd., Beijing, China]. Proteins was then loaded into gels for $10 \%$ SDS-PAGE and transferred to a polyvinylidene difluoride membrane in an ice bath (constant voltage of $100 \mathrm{~V}$ for $2 \mathrm{~h}$ ). Subsequent to blocking by $5 \%$ skimmed milk for $1 \mathrm{~h}$ at room temperature, the following primary antibodies were added: Rabbit anti-human polyclonal OPN (1:1,000; cat. no. ab8448) and rabbit anti-human $\beta$-actin (1:5,000; cat. no. ab129348). Following the incubation of the primary antibodies at $4^{\circ} \mathrm{C}$ overnight, horseradish peroxidase-conjugated goat anti-rabbit IgG secondary antibodies (1:3,000; cat. no. ab6721) were added prior to incubation at room temperature for $1 \mathrm{~h}$. All antibodies were purchased from Abcam. Between incubations, the membranes were extensively washed five times (5 min each) with PBS Tween 20. Finally, the membrane was developed by an enhanced chemiluminescence reagent (cat. no. ab65623; Abcam). The developed film was then scanned and analyzed by Image Lab 3.0 software (Bio-Rad Laboratories, Inc., Hercules, CA, USA). $\beta$-actin was used as an internal control to calculate the relative expression of OPN.

MTT assay. In order to examine cell viability, the transfected MH7A cells were seeded in 96-well plates at an approximate density of $2 \times 10^{3}$ cells/well, with three replicates for each well. At 24, 48 and 72 h, $20 \mu 15 \mathrm{~g} / 1 \mathrm{MTT}$ was added into each well, after which $150 \mu \mathrm{l}$ dimethyl sulfoxide was added to resolve the purple crystals. Subsequent to incubation for $4 \mathrm{~h}$ at $37^{\circ} \mathrm{C}$, the absorbance of cells was measured at $490-\mathrm{nm}$ wavelength and the proliferation curves were plotted.

Statistical analysis. The SPSS version 18.0 software (SPSS, Inc., Chicago, IL, USA) was used to conduct statistical analysis. All the data are expressed as the mean \pm standard deviation, and a normality test was used. One-way analysis of variance was used to compare differences among multiple groups. When variance was homogenous, the least significant difference (LSD) and Student-Newman-Keuls (SNK) methods were used; otherwise, Tamhane's T2 or T3 methods were applied. $\mathrm{P}<0.05$ was considered to demonstrate a difference that was statistically significant.

\section{Results}

Expression of OPN mRNA and protein levels in the blood and joint fluid of patients. In order to detect the expression changes in OPN mRNA and protein levels, RT-qPCR and ELISA were performed, respectively. Compared with the control osteoarthritis patients, OPN mRNA expression was significantly increased in the blood and joint fluid of RA patients $(\mathrm{P}<0.01$; Fig. 1). In addition, compared with the control group, OPN protein was also markedly enhanced in the blood and joint fluid of RA patients ( $\mathrm{P}<0.05$; Fig. 2). These results indicated that OPN mRNA and protein levels were consistently increased in both the blood and joint fluid of RA patients, suggesting that OPN may serve key roles in the pathogenesis of RA.

Expression of miR-539 in the blood and joint fluid. In order to investigate the expression of miR-539 in the blood and joint 

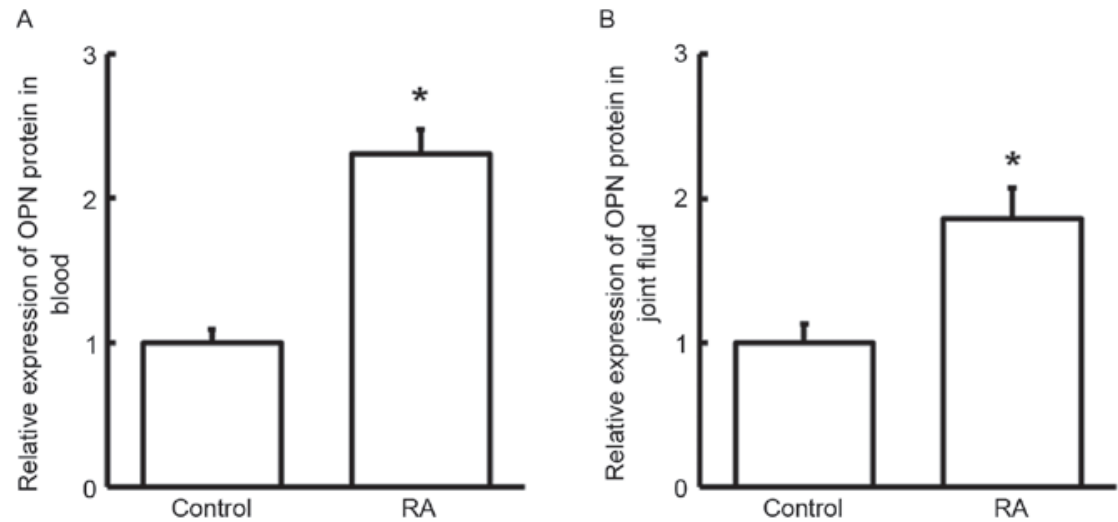

Figure 2. OPN protein expression in the (A) blood and (B) joint fluid of RA patients, examined by ELISA. Compared with osteoarthritis control patients, the expression of OPN protein was significantly increased in the blood and joint fluid of RA patients. "P<0.05 vs. control patients. OPN, osteopontin; RA, rheumatoid arthritis
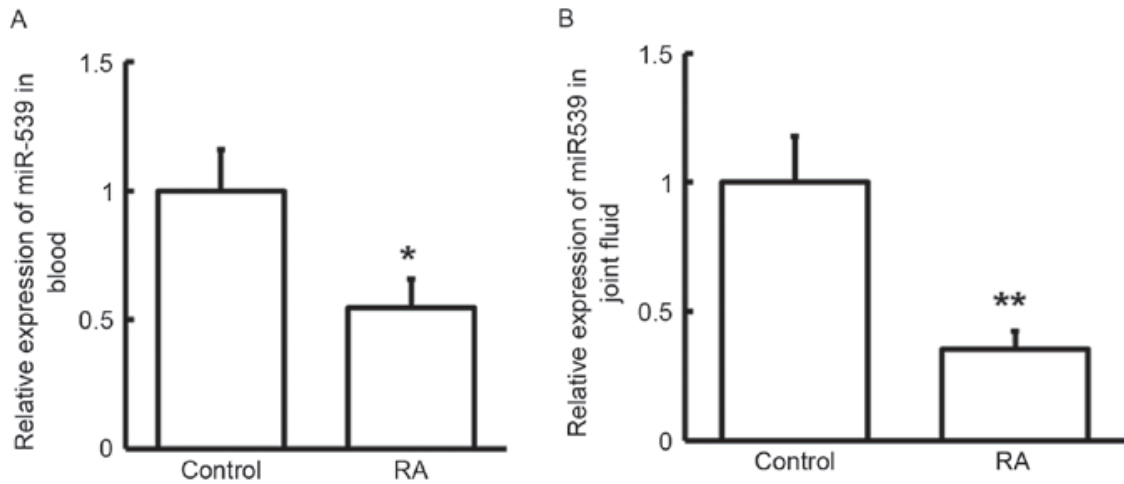

Figure 3. miR-539 expression in the (A) blood and (B) joint fluid of RA patients. Compared with the control patients, miR-539 expression was significantly decreased in the blood and joint fluid of RA patients. ${ }^{\mathrm{P}}<0.05$ and ${ }^{* * *} \mathrm{P}<0.01$ vs. control patients. RA, rheumatoid arthritis; miR, microRNA.

A
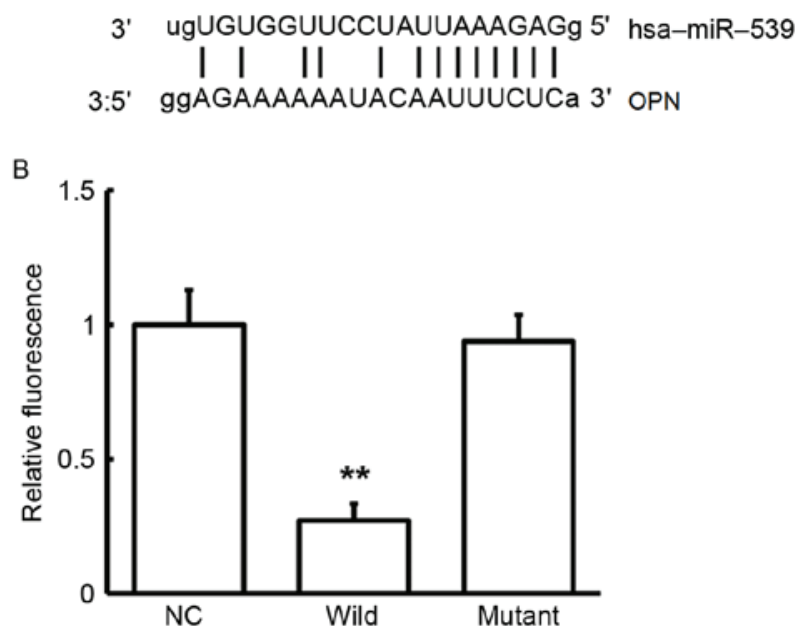

Figure 4. Analysis of miR-539 binding with OPN. (A) 'Seed' region of miR-539 and complementary binding site in the 3'UTR of OPN mRNA. (B) Dual-luciferase assay was used to detect whether OPN was directly targeted by miR-536. Compared with the NC group, fluorescence was significantly lower in cells transfected with wild-type OPN 3'UTR plasmid. ${ }^{* *} \mathrm{P}<0.01$ vs. NC group. OPN, osteopontin; RA, rheumatoid arthritis; NC, negative control; UTR, untranslated region; miR, microRNA.

fluid, RT-qPCR was used. As shown in Fig. 3, miR-539 expression in the two samples was significantly downregulated in RA patients when compared with the control patients $(\mathrm{P}<0.05)$. These results indicated that miR-539 level was decreased in both the blood and joint fluid of RA patients.

OPN is directly targeted by miR-539. To determine whether OPN was directly targeted by miR-539, bioinformatics prediction was initially performed. It was observed that mR-539 was able to target OPN. The complementary binding site of miR-539 with OPN is shown in Fig. 4A. To further confirm this observation, a dual-luciferase assay was conducted following co-transfection of agomiR-539 and pMIR-REPORT plasmid. As shown in Fig. 4B, fluorescence was significantly downregulated in cells co-transfected with agomiR-539 and pMIR-REPORT OPN wild-type plasmid when compared with the negative control group $(\mathrm{P}<0.01)$. By contrast, there was no significant difference between the group co-transfected with agomiR-539 and pMIR-REPORT OPN mutant and the negative control group $(\mathrm{P}>0.05)$. The aforementioned results suggest that miR-539 regulates OPN expression through complementary binding to the 3'-UTR of OPN mRNA.

Effects of miR-539 on the proliferation of MH7A cells. To examine the effect of miR-539 on MH7A cells, the expression levels of miR-539 and OPN in MH7A cells were detected following transfection with agomiR-539. Next, MTT was applied to detect the alterations in MH7A cell 


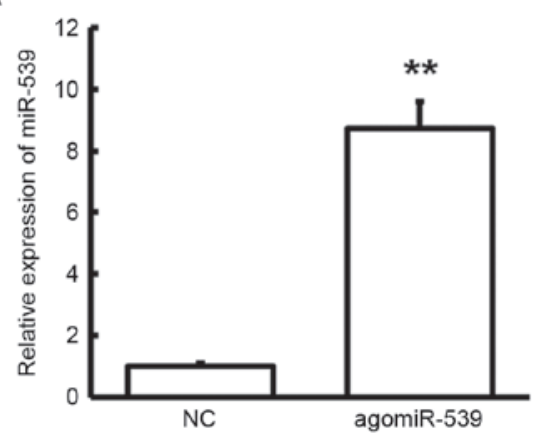

C

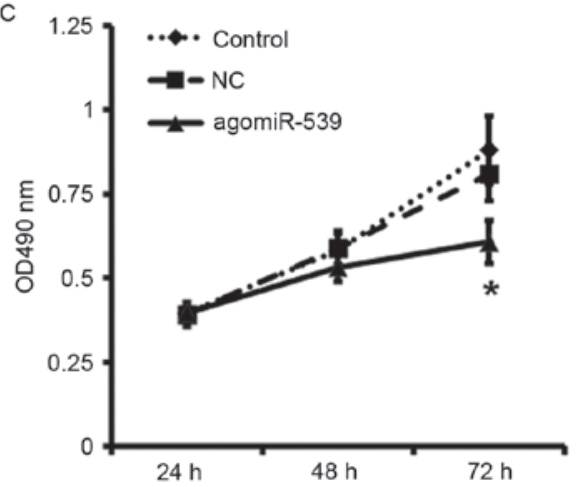

B

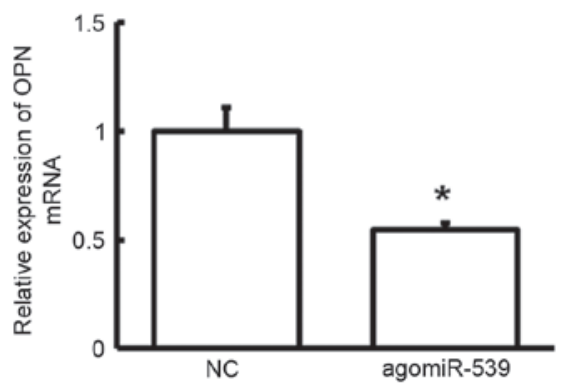

Figure 5. Effects of transfection with agomiR-539 on MH7A cells. (A) miR-539 expression was significantly increased in MH7A cells following transfection with agomiR-539. (B) OPN mRNA expression was significantly decreased in MH7A cells following transfection. (C) Compared with the control group, the proliferation was significantly inhibited in the agomiR-539 transfection group. Cells in control group were not transfected with any plasmid, while those in $\mathrm{NC}$ group were transfected with agomiR-NC plasmid. The control group was used to test whether transfection agent affected the viability of the cells in cell activity assay. ${ }^{\mathrm{P}}<0.05$ and ${ }^{* *} \mathrm{P}<0.01$ vs. NC group. OPN, osteopontin; RA, rheumatoid arthritis; NC, negative control; miR, microRNA; OD, optical density.

A

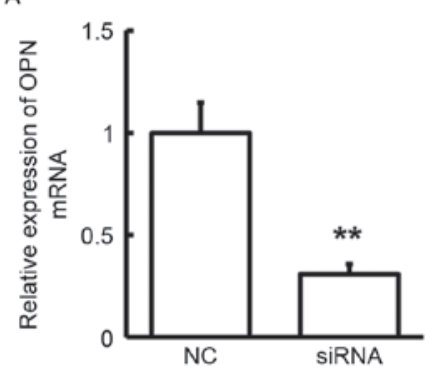

C

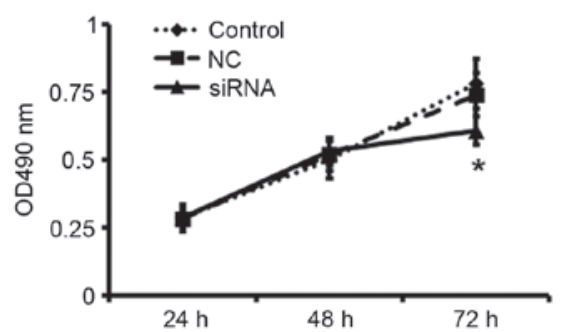

B

OPN
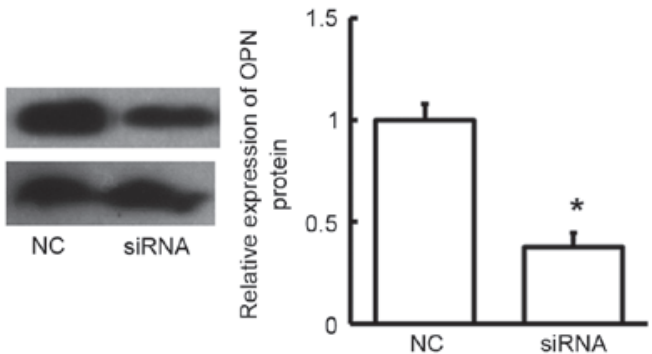

Figure 6. Effects of transfection with OPN siRNA on MH7A cells. (A) mRNA and (B) protein expression levels of OPN were significantly decreased in MH7A cells following transfection with OPN siRNA. (C) Compared with the control group, the proliferation was significantly inhibited in the OPN siRNA transfection group. ${ }^{*} \mathrm{P}<0.05$ and ${ }^{* *} \mathrm{P}<0.01$ vs. NC group. OPN, osteopontin; RA, rheumatoid arthritis; NC, negative control; miR, microRNA; OD, optical density.

proliferation. As shown in Fig. 5A, miR-539 expression was significantly increased in MH7A cells following transfection with agomiR-539 $(\mathrm{P}<0.01)$. At the same time, as shown in Fig. 5B, OPN mRNA expression was significantly decreased following transfection $(\mathrm{P}<0.01)$. Furthermore, compared with the control group, MH7A cell proliferation was significantly inhibited subsequent to transfection with agomiR-539 $(\mathrm{P}<0.05$; Fig. $5 \mathrm{C})$. These results indicate that miR-539 may 
inhibit the proliferation of MH7A cells through regulating OPN expression.

Effects of OPN siRNA on MH7A cells. To further validate the aforementioned effects of agomiR-539 through downregulation of OPN expression, RNA interference technology was used to downregulate OPN expression and then detect the cell proliferation by MTT assay. As shown in Fig. 6A and B, OPN expression in the mRNA and protein levels was significantly decreased following transfection with OPN siRNA in MH7A cells $(\mathrm{P}<0.05)$. In addition, the MTT assay results revealed that cell proliferation was significantly inhibited in the agomiR-539 transfection group (Fig. 6C). These results further confirm that downregulation of OPN may inhibit the proliferation of MH7A cells.

\section{Discussion}

In the present study, OPN expression was examined in the blood and joint fluid in RA patients, as well as the expression of the upstream regulator miR-539. In addition, the function and mechanism of miR-539 and OPN in the development of RA were preliminary discussed.

RA is a chronic and systemic autoimmune inflammatory disease with unknown etiology, and the clinical manifestation involves symmetric and multi-articular synovial arthritis, which is characterized by synovial tissue proliferation, angiogenesis and granulation tissue formation $(18,19)$. In the pathological processes of RA, the synovial inflammation reaction invades the cartilage and bone tissues to damage joint structure. In addition, the inflammatory reaction induces angiogenesis and promotes the proliferation of synovial cells, which further accelerate joint dysfunction and lead to joint deformity, stiffness and eventual loss of function (20). If patients are not diagnosed and treated at an early stage, treatment of the disease becomes challenging, leading to joint deformity and high disability rates reaching $60-70 \%$ (21). Therefore, it is important to clarify the pathogenesis and identify novel target genes for RA therapy.

OPN is a type of tumor-associated protein that was originally extracted from a malignant epithelial cell line. Through binding with its receptor CD44 and integrin, OPN promotes tumor cell adhesion, extracellular matrix degradation and cell apoptosis inhibition (22). It also promotes angiogenesis and inhibits immune reaction, which induces the invasion and metastasis of tumor cells (23). OPN is highly expressed in the articular cartilage, synovial fluid and synovium of osteoarthritis patients, while its expression has been positively correlated to the severity of the disease, indicating that OPN is closely associated with the development and progression of osteoarthritis $(24,25)$. In animal experiments, it was observed that OPN gene-deficient mice had a strong ability to resist collagen-induced arthritis (26). Furthermore, it was demonstrated that OPN was mainly expressed in the invaded articular cartilage pannus through in situ hybridization (27). All these findings indicated that OPN may serve important roles in the pathogenesis of RA. In the present study, OPN was highly expressed in the blood and joint fluid in RA patients, while OPN silencing suppressed the proliferation of MH7A cells, a human rheumatoid arthritis synovial cell line, indicating that OPN served important biological roles in RA.
To further investigate the regulatory mechanisms of OPN, the upstream regulators of OPN were predicted in the current study through bioinformatics methods. It was observed that miRNAs may inhibit OPN mRNA translation through degrading OPN mRNA (28). These regulatory mechanisms of miRNAs to upregulate or downregulate gene expression are involved in the development and progression of diseases $(29,30)$. According to the bioinformatics analysis, miR-539 was found to be a possible upstream miRNA regulating OPN. In addition, as observed in the earlier experiments, miR-539 was significantly decreased in the blood and joint fluid of RA patients. Considering the abnormally high expression of OPN in the blood and joint fluid of these patients, it was hypothesize that the downregulated expression of miR-539 may result in increased expression of OPN, which finally affects the biological properties of human synovial cells. To further examine the underlying molecular mechanism, the human rheumatoid arthritis synovial MH7A cell line was cultured in vitro and transfected with agomiR-539 by the liposome method. The cell proliferation was then analyzed by MTT assay. The results indicated that miR-539 suppressed the proliferation of MH7A cells and upregulated miR-539, inducing the downregulation of OPN. Further experiments through dual-luciferase assay identified that miR-539 was able to directly bind to the 3'UTR of OPN mRNA.

In conclusion, the results of the present study suggested that miR-539 regulates OPN expression through complementary binding to 3 -UTR. OPN is significantly increased in the blood and joint fluid of RA patients and is associated with the downregulation of miR-539. In the present study, the selected specimens were joint fluid and routine blood, which are easy to obtain. Therefore, miR-539 may potential in minimally invasive diagnoses of rheumatoid arthritis.

\section{Acknowledgements}

This study was supported by the Nanjing Health Youth Talent Project, Nanjing Department of Health (grant no. QRX11026), and the Science and Technology Project of Jiangsu Provincial Bureau of Traditional Chinese Medicine (grant no. YB2015124).

\section{References}

1. Goodman SM and Figgie MA: Arthroplasty in patients with established rheumatoid arthritis (RA): Mitigating risks and optimizing outcomes. Best Pract Res Clin Rheumatol 29: 628-642, 2015.

2. Canadian Agency for Drugs and Technologies in Health: Biologic Switching for Patients with Rheumatoid Arthritis: A Review of Clinical Effectiveness, Safety, and Guidelines. In: Rapid Response Reports: Summary with Critical Appraisal. Canadian Agency for Drugs and Technologies in Health, Ottawa, ON, 2015.

3. Sandoo A, Veldhuijzen van Zanten JJ, Metsios GS, Carroll D and Kitas GD: Vascular function and morphology in rheumatoid arthritis: A systematic review. Rheumatology (Oxford) 50: 2125-2139, 2011.

4. Lu Z and Zhong N: Internal medicine. People's Medical Publishing House, Beijing, 2008.

5. Holmdahl R (ed): The Hereditary Basis of Rheumatic Diseases. Birkhäuser Basel, Basel, 2006.

6. Churov AV, Oleinik EK and Knip M: MicroRNAs in rheumatoid arthritis: Altered expression and diagnostic potential. Autoimmun Rev 14: 1029-1037, 2015. 
7. Chen XM, Huang QC, Yang SL, Chu YL, Yan YH, Han L, Huang Y and Huang RY: Role of micro RNAs in the pathogenesis of rheumatoid arthritis: Novel perspectives based on review of the literature. Medicine (Baltimore) 94: e1326, 2015.

8. Murata K, Yoshitomi H, Furu M, Ishikawa M, Shibuya H, Ito H and Matsuda S: MicroRNA-451 down-regulates neutrophil chemotaxis via p38 MAPK. Arthritis Rheumatol 66: 549-559, 2014.

9. Jeanson-Leh L, Lameth J, Krimi S, Buisset J, Amor F, Le Guiner C, Barthélémy I, Servais L, Blot S, Voit T and Israeli D: Serum profiling identifies novel muscle miRNA and cardiomyopathy-related miRNA biomarkers in golden retriever muscular dystrophy dogs and duchenne muscular dystrophy patients. Am J Pathol 184: 2885-2898, 2014.

10. Chen YP, Jin X, Xiang Z, Chen SH and Li YM: Circulating MicroRNAs as potential biomarkers for alcoholic steatohepatitis. Liver Int 33: 1257-1265, 2013.

11. Hu H, Zhang Y, Cai XH, Huang JF and Cai L: Changes in microRNA expression in the MG-63 osteosarcoma cell line compared with osteoblasts. Oncol Lett 4: 1037-1042, 2012.

12. Xu SF, Zhao FT, Zhao H, Wang YL and Liu QF: Study of Effects of Direct Intra-articular Injection of Adenovirus-Fasl on Apoptosis of Synoviocytes in Adjuvant Arthritis Rats. Medical Recapitulate 18: 443-445, 2012.

13. Chen G, Zhang X, Li R, Fang L, Niu X, Zheng Y, He D, Xu R and Zhang JZ: Role of osteopontin in synovial Th17 differentiation in rheumatoid arthritis. Arthritis Rheum 62: 2900-2908, 2010.

14. Attur MG, Dave MN, Stuchin S, Kowalski AJ, Steiner G, Abramson SB, Denhardt DT and Amin AR: Osteopontin: An intrinsic inhibitor of inflammation in cartilage. Arthritis Rheum 44: 578-584, 2001.

15. Honsawek S, Tanavalee A, Sakdinakiattikoon M, Chayanupatkul $\mathrm{M}$ and Yuktanandana P: Correlation of plasma and synovial fluid osteopontin with disease severity in knee osteoarthritis. Clin Biochem 42: 808-812, 2009.

16. Kim KJ, Kwak J, Lee JH and Lee SS: Real-time qRT-PCR assay for the detection of miRNAs using bi-directional extension sequences. Anal Biochem 536: 32-35, 2017.

17. Livak KJ and Schmittgen TD: Analysis of relative gene expression data using real-time quantitative PCR and the 2(-Delta Delta C(T)) method. Methods 25: 402-408, 2001.

18. Chieng LO, Madhavan K and Vanni S: Pooled data analysis on anterior versus posterior approach for rheumatoid arthritis at the craniovertebral junction. Neurosurg Focus 38: E18, 2015.

19. Caplazi P, Baca M, Barck K, Carano RA, DeVoss J, Lee WP Bolon B and Diehl L: Mouse models of rheumatoid arthritis. Vet Pathol 52: 819-826, 2015.
20. Olumuyiwa-Akeredolu OO and Pretorius E: Platelet and red blood cell interactions and their role in rheumatoid arthritis. Rheumatol Int 35: 1955-1964, 2015.

21. Kerola AM, Kauppi MJ, Nieminen T, Rantalaiho V, Kautiainen H, Kerola T, Virta LJ, Pohjolainen T and Puolakka K: Psychiatric and cardiovascular comorbidities as causes of long-term work disability among individuals with recent-onset rheumatoid arthritis. Scand J Rheumatol 44: 87-92, 2015.

22. Lin Y and Yang-Yen HF: The osteopontin-CD44 survival signal involves activation of the phosphatidylinositol 3-kinase/Akt signaling pathway. J Biol Chem 276: 46024-46030, 2001.

23. Wang KX and Denhardt DT: Osteopontin: Role in immune regulation and stress responses. Cytokine Growth Factor Rev 19: 333-345, 2008

24. Gattorno M, Gregorio A, Ferlito F, Gerloni V, Parafioriti A, Felici E, Sala E, Gambini C, Picco P and Martini A: Synovial expression of osteopontin correlates with angiogenesis in juvenile idiopathic arthritis. Rheumatology (Oxford) 43: 1091-1096, 2004.

25. Gao SG, Li KH, Zeng KB, Tu M, Xu M and Lei GH: Elevated osteopontin level of synovial fluid and articular cartilage is associated with disease severity in knee osteoarthritis patients. Osteoarthritis Cartilage 18: 82-87, 2010.

26. Yumoto K, Ishijima M, Rittling SR, Tsuji K, Tsuchiya Y, Kon S, Nifuji A, Uede T, Denhardt DT and Noda M: Osteopontin deficiency protects joints against destruction in anti-type II collagen antibody-induced arthritis in mice. Proc Natl Acad Sci USA 99: 4556-4561, 2002.

27. Petrow PK, Hummel KM, Schedel J, Franz JK, Klein CL, Müller-Ladner U, Kriegsmann J, Chang PL, Prince CW, Gay RE and Gay S: Expression of osteopontin messenger RNA and protein in rheumatoid arthritis: Effects of osteopontin on the release of collagenase 1 from articular chondrocytes and synovial fibroblasts. Arthritis Rheum 43: 1597-1605, 2000.

28. Liu K, Liu S, Zhang W, Jia B, Tan L, Jin Z and Liu Y: miR-494 promotes cell proliferation, migration and invasion and increased sorafenib resistance in hepatocellular carcinoma by targeting PTEN. Oncol Rep 34: 1003-1010, 2015.

29. Lewis BP, Burge CB and Bartel DP: Conserved seed pairing, often flanked by adenosines, indicates that thousands of human genes are microRNA targets. Cell 120: 15-20, 2005.

30. Chen K and Rajewsky N: The evolution of gene regulation by transcription factors and microRNAs. Nat Rev Genet 8: 93-103, 2007. 